\title{
Gender-Based Differences in Abdominal Aortic Aneurysm Rupture: A Retrospective Study
}

\author{
Srikrishna Varun Malayala ${ }^{\mathrm{a}, \mathrm{d}}$, Ambreen Raza $^{\mathrm{b}}$, Rachana Vanaparthy ${ }^{\mathrm{c}}$
}

\begin{abstract}
Background: Annually, $5 \%$ of sudden deaths are due to abdominal aortic aneurysm (AAA) rupture. There is evidence suggesting that AAA ruptures have worse outcomes in females than males and the aneurysms rupture at a smaller size in females than in males. The United States Preventive Services Task Force (USPSTF) recommends a one-time ultrasound screening for males aged $65-75$ years who ever smoked. There is insufficient evidence to screen females aged 65 - 75 years who ever smoked though there is evidence suggesting that AAAs rupture at a smaller size and have worse outcomes in females. The objective of this study is to compare the characteristics, mortality and morbidity of ruptured AAAs in females and males.
\end{abstract}

Methods: This is a retrospective review of 117 patients from two teaching institutions over a period of 6 years. A total of 39 parameters were compared between males and females including demographic variables, comorbidities like hypertension, dyslipidemia, cardiovascular diseases; previous history of AAA; medications, characteristics of aneurysm, type of surgery and its outcome; postoperative complications and long-term survival.

Results: The overall incidence of AAA rupture was higher in males $(68 \%)$ than in females $(32 \%)$. Females die from AAA rupture at a later age. There was a significant difference in the size of AAA rupture between females (mean $=7.4 \mathrm{~cm}$, standard deviation $(\mathrm{SD})=$ $2.0)$ and males (mean $=8.2 \mathrm{~cm}, \mathrm{SD}=1.8 ; \mathrm{P}=0.04)$. The probability to undergo surgery for ruptured AAA was significantly lower for females as compared to males $(\mathrm{P}=0.03)$. Females had higher overall mortality $(\mathrm{P}=0.001)$, postoperative mortality $(\mathrm{P}=0.02)$, higher length of intensive care unit (ICU) stay, incidence of postoperative complications, use of vasopressors and use of ventilator.

Manuscript submitted October 25, 2020, accepted October 31, 2020

Published online December 18, 2020

aDepartment of Medicine, Temple University Health System, Philadelphia, PA, USA

bepartment of Medicine, Bristol Hospital, Farmington, CT, USA

'Department of Pulmonology, Oregon Health and Science University, Portland, OR, USA

${ }^{\mathrm{d} C}$ Corresponding Author: Srikrishna Varun Malayala, Department of Medicine, Temple University, Philadelphia, PA 19111, USA.

Email: Srikrishna.V.Malayala@tuhs.temple.edu

doi: https://doi.org/10.14740/jocmr4376
Conclusions: Using a similar threshold of size of AAA for elective surgery for both males and females might not be appropriate. Further population-based studies are needed to warrant AAA screening for high-risk females owing to the higher morbidity and mortality.

Keywords: Aneurysm; Females; Mortality; AAA; Rupture; Ultrasound; Screening

\section{Introduction}

Abdominal aortic aneurysms (AAA) affect males to females at the ratio of 4:1, but females are known to have worse outcomes [1]. Available literature suggests that ruptured aneurysms occur in females more frequently and also at smaller diameters compared to males [1]. The prevalence of AAAs with diameter larger than $2.9 \mathrm{~cm}$ in males ranges from $1.9 \%$ to $18.5 \%$ which is much higher than females (0-4.2\%) [2]. Females with AAAs usually present late, grow rapidly than males, and also have a four-time higher risk of rupture at a diameter $5.0-5.9 \mathrm{~cm}$ than males [3]. The average annual risk of rupture of AAA with $\geq 6$ $\mathrm{cm}$ is $14.1 \%$ in males and $22.3 \%$ in females. The mortality of AAA rupture is about $50 \%$ [4].

In 2017, total deaths due to AAA were about 9,928, which was up from 2,600 in 2013 [5, 6]. Surgical intervention either by endovascular repair (EVAR) or open repair is offered for aneurysms $\geq 5.5 \mathrm{~cm}$ in both males and females. Rupture of AAA is a medical emergency and is associated with a very high prehospitalization mortality [7]. Some reports also suggest that females who received EVAR or open repair were more likely to have a higher postoperative mortality than males. Differences in diagnosis and treatment rates or inherent anatomical dissimilarities and lack of screening for females seem to be the reasons for higher mortality rate in females [5].

Ultrasound has $90 \%$ sensitivity and $100 \%$ specificity to diagnose AAA and has been the standard screening tool for AAA. The 2019 United States Preventive Services Task Force (USPSTF) guidelines recommend one-time screening with ultrasonography in males aged $65-75$ years who had ever smoked. When it comes to screening guidelines in females, USPSTF concludes that there is insufficient evidence to screen females aged 65 - 75 years who had ever smoked [8]. These recommendations have been consistent for many years even after multiple studies suggesting higher mortality in females as illustrated above. While further population-based studies are 
needed to address the guidelines related to screening; with this study, we attempt to specifically study the AAA ruptures and subsequent outcomes in females.

\section{Materials and Methods}

\section{Objectives}

The objective of the study was to compare the outcomes of ruptured AAAs between males and females. We also compared the patients presenting with the ruptured AAAs, the characteristics of the aneurysms itself and postoperative course between males and females.

\section{Sample}

The study was nested as a multi-center retrospective study and the sample was obtained from two community-based sister hospitals located in a metro city in Western New York. Over a period of 6 years, all the patient charts admitted with a diagnosis of AAA in the two hospitals were reviewed. The data were collected through a retrospective review of paper charts and electronic medical records. Approval was obtained from the Institution's Review Board (IRB) of the hospital system.

The inclusion criteria for the study were the patients who presented with a rupture of the aneurysm. The patients who were admitted for elective repair were excluded.

\section{Variables}

Demographic variables like age and gender, patient's comorbidities (hypertension, dyslipidemia, diabetes mellitus, cardiovascular diseases), previous history of AAA (size at previous diagnosis, previous attempt to repair and history of rupture), current use of cardio-protective medications (statins, aspirin, clopidogrel and beta-blockers), the characteristics of aneurysm (size, involvement of iliac arteries) were noted and compared between males and females. They were labeled as smokers if they smoked at least 100 cigarettes in their lifetime.

The computed tomography (CT) scan findings at the time of presentation to the hospital were used to identify the ruptured aneurysms, their size (in centimeters), location (supra or infrarenal) and iliac artery involvement.

The hospital course including length of stay in intensive care unit (ICU) and hospital, incidence and type of surgery, immediate postoperative complications like ventilator dependent respiratory failure, hypovolemic shock, acute renal failure, and myocardial infarction were reviewed. The immediate postoperative mortality and overall mortality with or without surgery were noted. The long-term survival of the discharged patients was identified using the SSN database. Patients discharged alive were followed for a period of 2 years. Date of death were procured from https://ladmf.ntis.gov (SSN database).

A total of 39 parameters were compared between males and females. The patient data were kept confidential and the only primary investigators had access to the data through a personal access key.

\section{Statistical analysis}

SPSS version 26 was used for analysis of the study. We analyzed between-group differences on demographic and baseline characteristics by using Chi-square tests for categorical variables and $t$-tests for continuous variables. The predictors of mortality were projected based on a logistic regression model, adjusting for age, hypertension, major comorbidities, smoking status and use of preventive medications. We took the P value as less than 0.05 for statistical significance. Kaplan-Meier survival curve analysis was used to analyze the long-term survival of these patients (2-year mortality).

\section{Results}

A total of 1,538 patients were hospitalized to either hospital with a diagnosis of AAA. About $92 \%$ of the samples $(1,417$ patients) were excluded and 117 patients were included for final analysis (Fig. 1) as the sample was restricted to ruptured aneurysms only. Out of the 117 patients, 79 were male $(67.6 \%)$ and 38 were female patients (32.4\%) admitted with an AAA rupture.

Table 1 demonstrates the demographic variables of the sample, and $100 \%$ of the samples were Caucasian. Obesity was more prevalent in the male patients with a significant statistical difference $(\mathrm{P}=0.02)$, but there was no difference in the distribution of tobacco use, hypertension, other major cardiovascular comorbidities or use of any cardio-protective medications.

Gender was identified as an independent predictor of age of rupture after adjusting the effects of hypertension, comorbidities, smoking, use of medications and previous history of aneurysms $(\mathrm{P}=0.005)$. The mean age of rupture in males was 75.8 years (standard deviation $(\mathrm{SD})=10$ ); and in females, it was 82.4 years $(\mathrm{SD}=8.6)$. We evaluated the age specific incidence of the aneurysm ruptures and it was noted that $65.8 \%$ of the male patients were under 85 years age and $65.7 \%$ of the female patients were over 85 years old (Fig. 2).

Table 2 describes the characteristics of the ruptured aneurysms in male and female patients. There was no difference in the pattern of the location of the rupture, whether they are suprarenal, infrarenal or both, whether they are located on the left, right or both sides. There were 75 males $(94.9 \%)$ and 34 females $(89.5 \%)$ with an infrarenal aneurysm. However, there was a significant difference in the size of the aneurysm rupture between the two groups. Female patients tend to have a significantly smaller size of aneurysm at the time of rupture (mean $=7.46 \mathrm{~cm}, \mathrm{SD}=2.09$ ) as compared to the males (mean $=8.23 \mathrm{~cm}, \mathrm{SD}=1.84 ; \mathrm{P}=0.04)$. About $58 \%$ of the male patients had a known previous diagnosis of AAA and $29 \%$ of them had their aneurysms repaired on an elective basis. In the female patients, AAA was a known diagnosis in only $50 \%$ of 


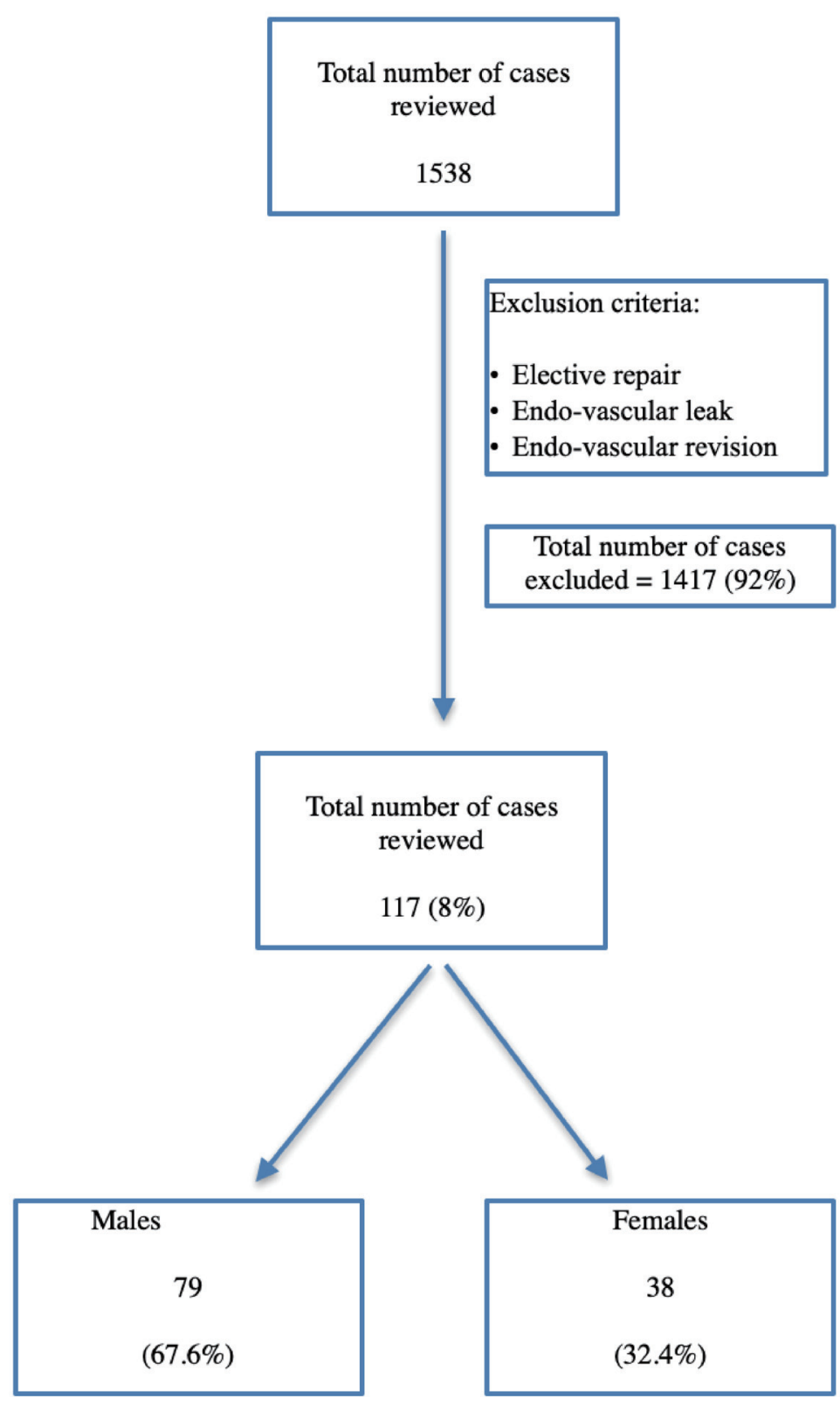

Figure 1. Inclusion and exclusion criteria of the study.

the cases and surgical repair was performed only in $10.5 \%$ of the cases. At their previous diagnosis, the mean size of the aneurysm was significantly smaller in males $(4.0 \mathrm{~cm}, \mathrm{SD}=3.3)$, as compared to the females $(5.0 \mathrm{~cm}, \mathrm{SD}=2.6 ; \mathrm{P}=0.03)$. The AAA was found in either routine screening or as an incidental finding.

Table 3 explains the characteristics describing hospital course and mortality of the ruptured AAAs and the postoperative complications. The overall mortality (irrespective of surgery or not) was significantly higher in women $(68.4 \%)$ as compared to men $(31.6 \%), \mathrm{P}<0.001$. The postoperative mortality was also higher in females which is $50 \%$ versus $21.2 \%$ in males, $\mathrm{P}=0.05$. The overall mortality and postoperative mor- tality were adjusted for tobacco use, age, major comorbidities and use of cardio-protective medications using a logistic regression model.

Out of the 79 male patients in the sample, 74 of them $(93.7 \%)$ had an operative management of the ruptured AAA as compared to only 24 of the 38 females $(63.2 \%), P=0.03$. Endovascular repair of the ruptured AAA was performed more frequently in both males and females (72.2\% vs. $42.1 \%)$ than open repair, $\mathrm{P}<0.01$. The postoperative complications (ventilator dependent respiratory failure, requirement of vasopressors and other unexpected complications like blood loss, renal failure, etc.) tend to happen more frequently in females as compared to males at a statistically significant level $(\mathrm{P}<$ 
Table 1. Demographic Characteristics of the Male and Female Patients Admitted With Abdominal Aortic Aneurysm (AAA) Rupture

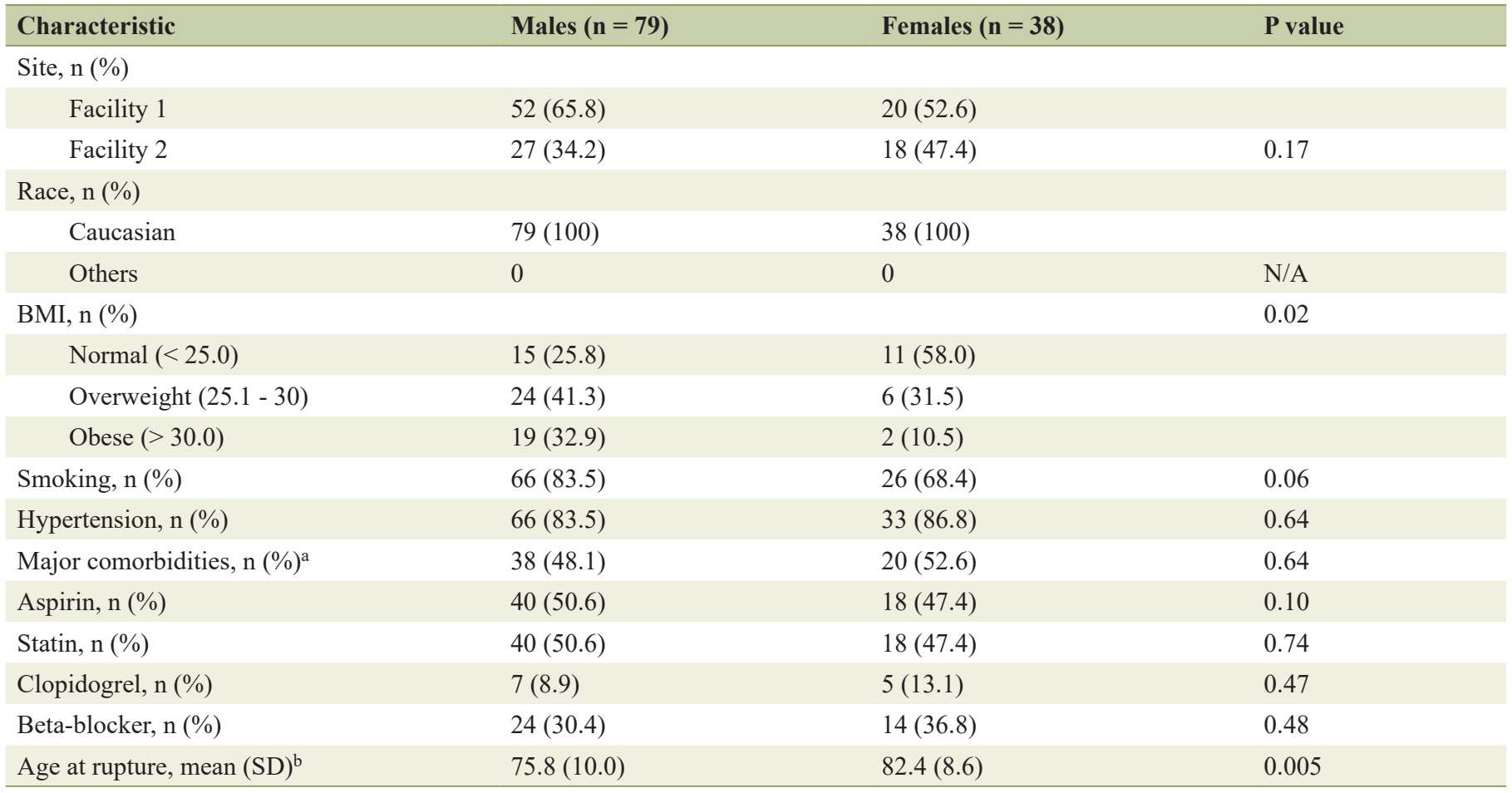

aMajor comorbidities are defined as diabetes mellitus, cardiovascular diseases including coronary artery, cerebrovascular and carotid artery diseases. ${ }^{b}$ Age was adjusted for BMI, smoking history, hypertension, comorbidities, use of aspirin, statin, clopidogrel, beta-blockers, previous history of aneurysm, size and location of aneurysm. BMl: body mass index; SD: standard deviation.

$0.001)$ even when adjusted for age, comorbidities, tobacco use and use of medications. The length of stay in the ICU was significantly longer in females as compared to males (5.5 days versus 4.1 days) using the same model, $\mathrm{P}=0.02$.

Figure 3 shows the long-term survival of the patients discharged alive after the AAA repair. The available sample for this analysis was 27 patients; out of them 21 were male and six patients were female patients. Males survived an average of 11.0 months $(\mathrm{SD}=2.2)$ as compared to 9.3 months $(\mathrm{SD}=$ $2.9)$ in females, though not at a significant level $(\mathrm{P}=0.41)$.

\section{Discussion}

Cardiovascular disease is the number one cause of death for both men and women in the USA [3]. Traditionally, all the car-

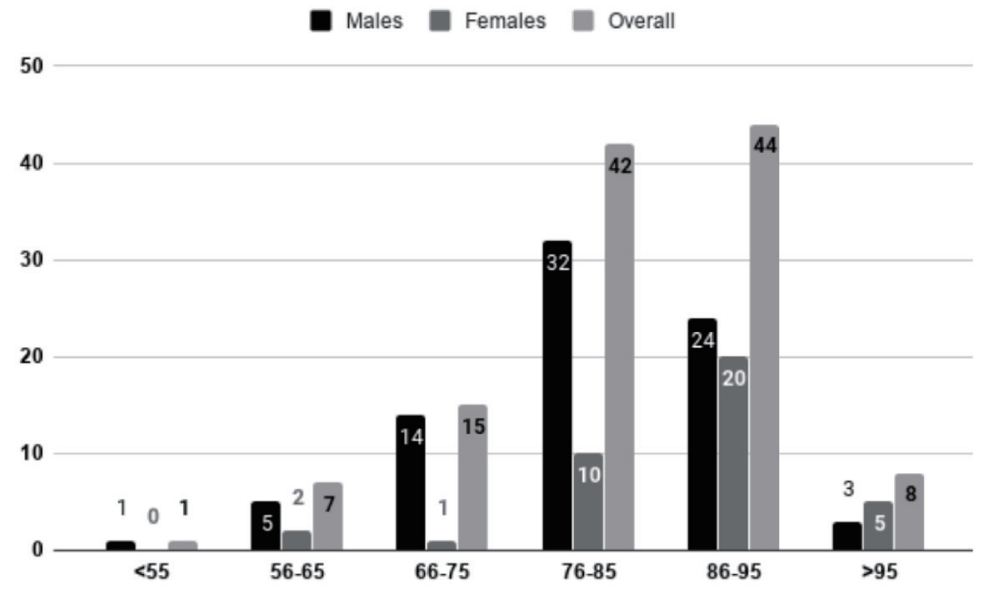

Figure 2. Age specific distribution of male and female patients admitted with abdominal aortic aneurysm (AAA). It was noted that $65.8 \%$ of the male patients admitted with AAA rupture were under 85 years of age and $65.7 \%$ of the female patients admitted for AAA rupture were more than 85 years old. 
Table 2. Characteristics of the Ruptured Abdominal Aortic Aneurysms (AAA) in the Male and Female Patients at Presentation, Based on the CT Scan Findings

\begin{tabular}{|c|c|c|c|}
\hline Characteristic & Males $(n=79)$ & Females $(\mathbf{n}=\mathbf{3 8})$ & P value \\
\hline Location of the aneurysm, n (\%) & & & 0.28 \\
\hline Infrarenal & $75(94.9)$ & $34(89.5)$ & \\
\hline Both & $4(5.1)$ & $3(7.9)$ & \\
\hline Involvement of iliac arteries, n (\%) & & & 0.42 \\
\hline Right & $9(11.4)$ & $4(10.5)$ & \\
\hline Both & $12(15.2)$ & $3(7.9)$ & \\
\hline None & $52(65.8)$ & $30(78.9)$ & \\
\hline Size of aneurysm at rupture $(\mathrm{cm})$, mean $(\mathrm{SD})$ & $8.23(1.84)$ & $7.46(2.09)$ & 0.04 \\
\hline Known history of AAA repair, n (\%) & $46(58.2)$ & $19(50)$ & 0.34 \\
\hline
\end{tabular}

CT: computerized tomography; SD: standard deviation.

diovascular diseases were considered as "men's diseases." Our study concludes that there was a significant effect of gender on the age of death from AAA rupture, and there was a significant difference in the size of AAA rupture between males and females. Women tend to present at an advanced age and have a smaller size of the ruptured aneurysm though the overall incidence of AAA rupture was higher in males. The probability of undergoing surgery for ruptured AAA was significantly lower for women. Female gender was also identified as an independent predictor of longer length of ICU stay, higher incidence of postoperative complications, more frequent use of vasopres- sors and ventilators.

There are a few previous studies which showed the disparities of risks and outcomes of AAAs in males and females, and our study adds to the literature that even ruptured aneurysms tend to fare worse in females. Females are usually protected from the development of AAAs, but behave aggressively with faster growth, frequency of rupture, and higher mortality rate [5].

One hundred percent of the patients included in our study were Caucasian. AAAs are commonly seen in Caucasian population, and few studies have reported differences in the

Table 3. Characteristics Describing the Hospital Course and Mortality of the Ruptured Abdominal Aortic Aneurysms in the Male and Female Patients

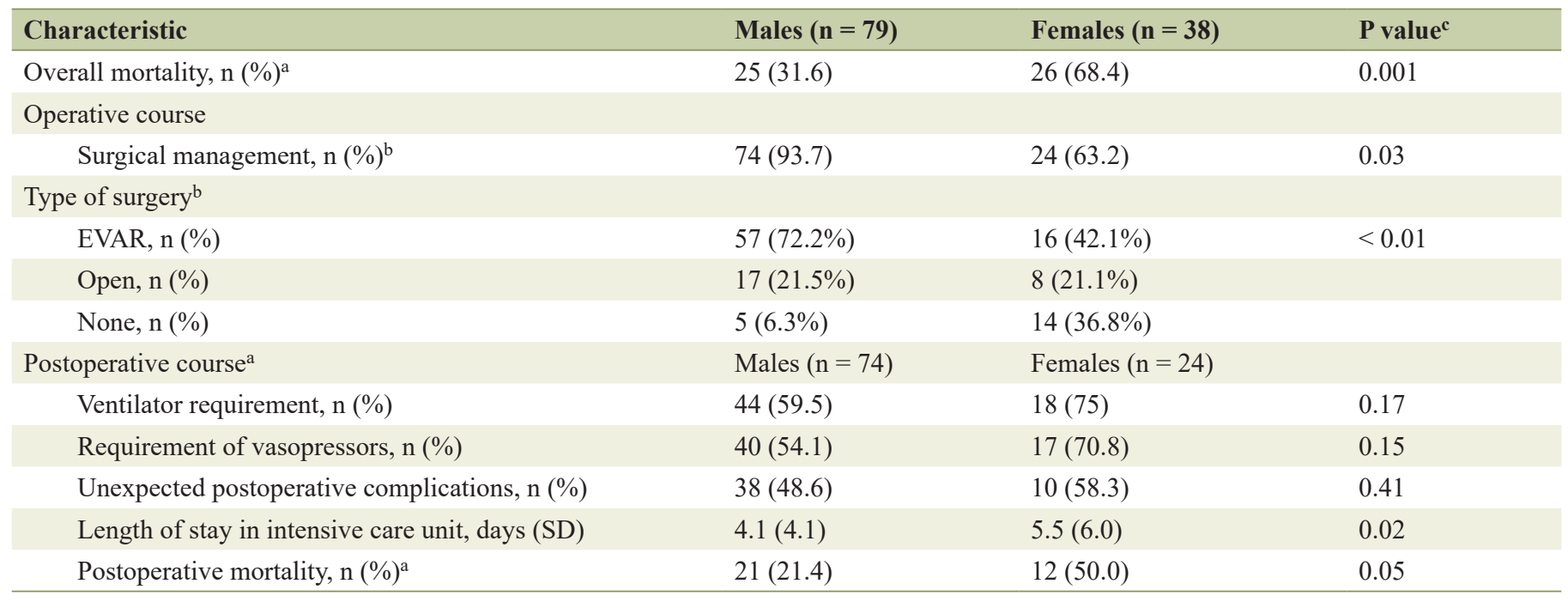

${ }^{a}$ Adjusted for tobacco use, age, size at presentation, major comorbidities and use of cardio-protective medications. ${ }^{\mathrm{b}}$ The incidence of surgery was adjusted for age, major comorbidities and size at presentation. ${ }^{\top}$ The overall mortality was still at a statistically significant level of less than 0.05 even for the unadjusted sample. However, the postoperative mortality for the unadjusted sample was 0.26 as compared to 0.05 for the adjusted sample. 


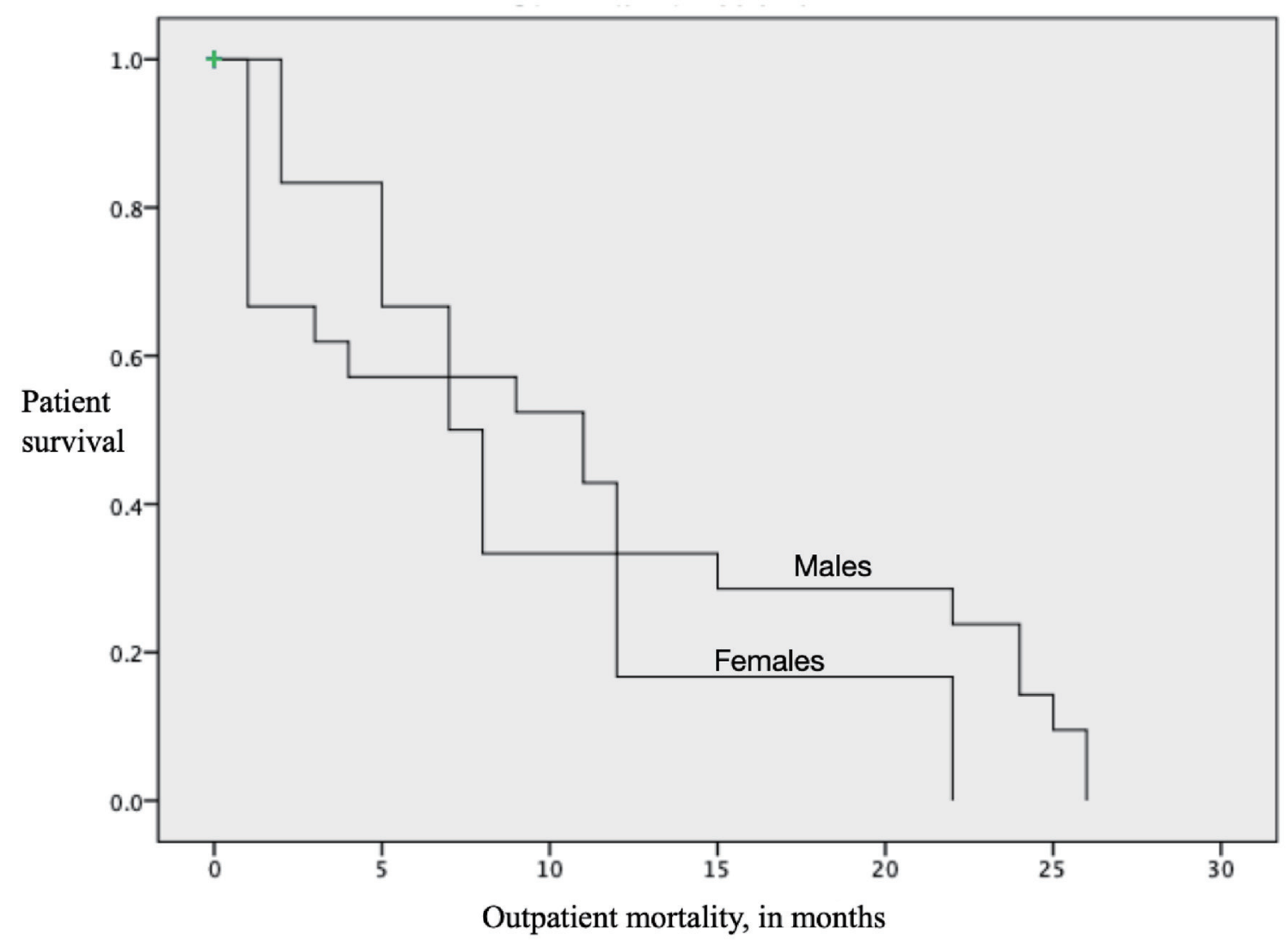

Figure 3. Long-term (over 2 years) survival of the patients discharged alive after AAA repair. $X$ axis represents the months of survival after an emergent abdominal aortic aneurysm (AAA) repair. $Y$ axis is the survival of the patients on a scale of 1 to 0 . Males survived an average of 11.0 months ( $S D=2.2$ months) as compared to 9.3 months in the females $(S D=2.9$ months, $P=$ 0.41). The total sample available for the analysis was 27 patients, 21 males and six females.

development of AAA in Caucasians and African American populations [9]. The number of females in the sample is also smaller as compared to males (38 versus 79 ), reflecting the prevalence pattern of AAAs. Out of 117 patients included in the study, $92(78.6 \%)$ of them have at least smoked 100 cigarettes in their lifetime. Smoking and genetics play a key role in the development of AAA [10]. Among all the cardiovascular diseases, AAA has the strongest association with smoking, precisely current smoking. In a study by Ulug et al on the prevalence of screening-detected AAAs in women, it was found that smoking had a greater impact on prevalence of AAA in females than in males [11]. As seen in our study, there is also a strong correlation between obesity with high waist circumference and AAA. This is probably due to the release of adipokines and obesity-induced aortic inflammation leading to weakening of the vessel and further aneurysmal formation [12]. We wanted to neutralize the effect of current use of medications on AAA outcomes as there is evidence suggesting that use of cardio-protective medications like aspirin, clopidogrel, statins and beta blockers have protective effects against AAA [13-16]. Ninety-three percent of our sample presented with infrarenal aneurysm, which is consistent with the data that $85 \%$ of the AAAs are infrarenal [17]. Though estrogen has a protective effect in the development of AAA in females, hormonal replacement therapy increases the risk of AAA development after menopause [18]. Overall, the development of AAA, its progression and outcome are a combination of genetic predispositions and environmental factors [10].

In our sample, mean diameter at the time of rupture in males is $8.23 \mathrm{~cm}$ with SD of 1.84 , while that of females is $7.46 \mathrm{~cm}(\mathrm{SD}=2.09)$. Few authors believe that discrepancies in geometric and bio-mechanical properties are the reasons for faster growth of AAA and increased rate of rupture than that of males in spite of low prevalence. Females also exhibit higher proportion of aneurysms with high peak wall stress [19]. In males, the average infrarenal diameter of aorta at the age of 65 years or more is $2.02 \mathrm{~cm}$, whereas in females it is $1.75 \mathrm{~cm}$ [11]. The intuitive thought for smaller diameters of AAA in females when compared to males is that females inherently have smaller aortas [19]. These innate differences could call for gender specific guidelines in screening and elective surgical repair of AAAs in females.

AAA is usually asymptomatic and diagnosis is usually made as an incidental finding on imaging for other medical complaints [20]. Sixty-five patients (55.5\%) in our sample had the previous diagnosis of AAA who were diagnosed as part of routine screening or as an incidental finding on imaging for 
other medical illnesses. Few studies suggest that non-specific inflammatory markers like D-dimer levels are elevated in patients with AAA, but there are no other diagnostic and prognostic markers for the diagnosis of AAA, and there is also no drug therapy to limit the progression of AAA [20-22]. The only effective way of diagnosis and follow-up of the AAA is abdominal ultrasound. No repeat ultrasound is recommended if the AAA is less than $3 \mathrm{~cm}$, but if the initial size is $3-4 \mathrm{~cm}$, an ultrasound is recommended every 2 - 3 years; and for the AAA sized $4-5.5 \mathrm{~cm}$, ultrasound is recommended every 6 months to 1 year [20].

Fifty percent of the females in our sample who had a previous diagnosis of AAA had a mean diameter of $5 \mathrm{~cm}$. Considering the worse outcomes in females with higher mortality and delayed age at presentation, a threshold of $5.5 \mathrm{~cm}$ might be too high for females though this has been the recommended size of elective repair in males [23]. The current indications for elective AAA repair include diameter of $5.5 \mathrm{~cm}$ for a patient with symptomatic AAA (irrespective of the size), and rapid expansion like $1 \mathrm{~cm}$ in 1 year irrespective of the size. Though elective AAA repair is associated with decreased morbidity and mortality, overall operative mortality and long-term survival mainly depend on the patient's age and other risk factors. Hence, the decision to perform an elective AAA repair must weigh the patient's risk of rupture depending on AAA diameter against the individual risk of surgery [24]. Endovascular repair $(72.2 \%$ and $42.1 \%)$ for ruptured AAA is performed more frequently than open repair $(21.5 \%$ and $21.1 \%)$ in both males and females [25]. In spite of advanced ICU and techniques for repair, mortality still remains high following repair of ruptured AAA [5]. We adjusted our sample for age, cardio-protective medications and comorbidities and still found that the postoperative complications, overall mortality and morbidity remain higher in females.

Postoperative complications tend to happen more commonly in females as compared to males at a statistically significant level $(\mathrm{P}<0.0001)$ when adjusted for age, comorbidities, tobacco use and use of medications. Hence, very close postprocedural surveillance and prompt correction of complications are required to avoid fatal outcomes [26]. Twenty-four out of 38 female patients had undergone surgical repair, either EVAR or open repair. Of these, $18(75 \%)$ patients required ventilator, $17(70.8 \%)$ required vasopressors and $10(58.3 \%)$ had other unexpected postoperative complications like blood loss, renal failure, etc. Females (12 out of 24, 50\%) who received EVAR or open repair have postoperative complications and are more likely to die than males. Differences in diagnosis and treatment rates or inherent anatomical dissimilarities and lack of screening for females are the reasons for high mortality rate in females after EVAR or open repair [5]. Hence, there is a need to focus on improving ruptured AAA outcomes in females with a repair at a smaller size and earlier age. EVAR has been identified as one of the biggest determinants in ruptured AAA outcomes. However, with this study we could not study the outcomes of EVAR versus open repair as the sample size was too small.

Gender-based differences in outcomes and sex specific guidelines have been identified and studied in multiple scenarios including cholesterol levels, use of prophylactic medi- cines and coronary artery disease screening and management [27-30]. In 2017, USPSTF considered a revised draft plan for AAA screening including asymptomatic males and females. If this plan is implemented, it is possible that there may be reduction in mortality disparity between males and females with ruptured AAA [5].

\section{Limitations}

Our study is prospective and single-centered. It lacks external validity required to support widespread changes in practice. We compared smoking, hypertension and other medications but we missed comparing covariates like family history and age of menopause in the retrospective review. Our findings could be considered hypothesis generating, perhaps serving as the basis for a larger study. While we were able to come to a conclusion that ruptures of AAA have worse outcomes in females as compared to males, we do realize that these results need reinforcements from other multi-center and population-based studies to comment on the screening guidelines. Also, the number of females in the sample is less than males (38 versus 79); this might reflect the overall prevalence of AAAs in females but limited a lot of analyses. For example, we could not study the outcomes of EVAR vs. open repair which has been identified as a significant predictor of outcome.

\section{Conclusions}

With the findings from our study, we call for further studies to suggest the importance of screening for AAAs in high-risk women and the importance of formulating "sex-specific" management guidelines of AAA. Routine screening in men of age 65 years and over has significantly reduced the mortality from ruptured AAA. Nearly one-third of the patients who present with ruptured AAA are women, and they do have a very high mortality contributing to a large proportion of deaths from AAA [11].

\section{Acknowledgments}

We acknowledge Dr. Paul Anain, Vascular Surgery, Sisters of Charity Hospital for his valuable support and feedback regarding the manuscript.

\section{Financial Disclosure}

None to declare.

\section{Conflict of Interest}

There are no conflicts of interest reported by any of the authors. 


\section{Informed Consent}

Not applicable.

\section{Author Contributions}

Srikrishna V Malayala: formulating study design, data collection, statistical analysis and formulating manuscript; Ambreen Raza: data collection, writing the manuscript; Rachana Vanaparthy: statistical analysis, literature review, and writing the manuscript.

\section{Data Availability}

The data supporting the findings of this study are available from the corresponding author upon reasonable request. Any inquiries regarding supporting data availability of this study should be directed to the corresponding author.

\section{References}

1. O'Donnell TFX, Verhagen HJ, Pratesi G, Pratesi C, Teijink JAW, Vermassen FEG, Mwipatayi P, et al. Female sex is associated with comparable 5-year outcomes after contemporary endovascular aneurysm repair despite more challenging anatomy. J Vasc Surg. 2020;71(4):11791189.

2. Grootenboer N, Bosch JL, Hendriks JM, van Sambeek MR. Epidemiology, aetiology, risk of rupture and treatment of abdominal aortic aneurysms: does sex matter? Eur J Vasc Endovasc Surg. 2009;38(3):278-284.

3. Ash JL, Khan KD, Syed MH. Abdominal aortic aneurysms in women: the debate continues. Endovascular Today. $2018 ; 1$.

4. Brown PM, Zelt DT, Sobolev B. The risk of rupture in untreated aneurysms: the impact of size, gender, and expansion rate. J Vasc Surg. 2003;37(2):280-284.

5. Stuntz M, Audibert C, Su Z. Persisting disparities between sexes in outcomes of ruptured abdominal aortic aneurysm hospitalizations. Sci Rep. 2017;7(1):17994.

6. Spark JI, Baker JL, Vowden P, Wilkinson D. Epidemiology of abdominal aortic aneurysms in the Asian community. Br J Surg. 2001;88(3):382-384.

7. Keisler B, Carter C. Abdominal aortic aneurysm. Am Fam Physician. 2015;91(8):538-543.

8. U. S. Preventive Services Task Force, Owens DK, Davidson KW, Krist AH, Barry MJ, Cabana M, Caughey AB, et al. Screening for abdominal aortic aneurysm: US preventive services task force recommendation statement. JAMA. 2019;322(22):2211-2218.

9. Yii MK. Epidemiology of abdominal aortic aneurysm in an Asian population. ANZ J Surg. 2003;73(6):393-395.

10. Carino D, Sarac TP, Ziganshin BA, Elefteriades JA. Abdominal Aortic Aneurysm: Evolving Controversies and Uncertainties. Int J Angiol. 2018;27(2):58-80.
11. Ulug P, Powell JT, Sweeting MJ, Bown MJ, Thompson SG, Group SC. Meta-analysis of the current prevalence of screen-detected abdominal aortic aneurysm in women. Br J Surg. 2016;103(9):1097-1104.

12. Wang L, Djousse L, Song Y, Akinkuolie AO, Matsumoto C, Manson JE, Gaziano JM, et al. Associations of Diabetes and Obesity with Risk of Abdominal Aortic Aneurysm in Men. J Obes. 2017;2017:3521649.

13. Wemmelund H, Jorgensen TM, Hogh A, Behr-Rasmussen C, Johnsen SP, Lindholt JS. Low-dose aspirin and rupture of abdominal aortic aneurysm. J Vasc Surg. 2017;65(3):616-625 e614.

14. Liu O, Jia L, Liu X, Wang Y, Wang X, Qin Y, Du J, et al. Clopidogrel, a platelet P2Y12 receptor inhibitor, reduces vascular inflammation and angiotensin II inducedabdominal aortic aneurysm progression. PLoS One. 2012;7(12):e51707.

15. Salata K, Syed M, Hussain MA, de Mestral C, Greco E, Mamdani M, Tu JV, et al. Statins reduce abdominal aortic aneurysm growth, rupture, and perioperative mortality: a systematic review and meta-analysis. J Am Heart Assoc. 2018;7(19):e008657.

16. Chun AS, Elefteriades JA, Mukherjee SK. Do beta-Blockers Really Work for Prevention of Aortic Aneurysms?: Time for Reassessment. Aorta (Stamford). 2013;1(1):4551.

17. Studzinska D, Rudel B, Polok K, Lewandowski K, Studzinski K, Gajdosz A, Oo A, et al. Infrarenal versus suprarenal abdominal aortic aneurysms: comparison of associated aneurysms and renal artery stenosis. Ann Vasc Surg. 2019;58:248-254 e241.

18. Boese AC, Chang L, Yin KJ, Chen YE, Lee JP, Hamblin MH. Sex differences in abdominal aortic aneurysms. Am J Physiol Heart Circ Physiol. 2018;314(6):H1137H1152.

19. Lo RC, Schermerhorn ML. Abdominal aortic aneurysms in women. J Vasc Surg. 2016;63(3):839-844.

20. Moxon JV, Parr A, Emeto TI, Walker P, Norman PE, Golledge J. Diagnosis and monitoring of abdominal aortic aneurysm: current status and future prospects. Curr Probl Cardiol. 2010;35(10):512-548.

21. Golledge J. Assessment of plasma D-dimer as a diagnostic and prognostic aid for abdominal aortic aneurysm. Acute care testing.org. July 2011. https://acutecaretesting.org/ en/articles/assessment-of-plasma-d-dimer-as-a-diagnostic-and-prognostic-aid-for-abdominal-aortic-aneurysm.

22. Eckstein HH, Maegdefessel L. Linking obesity with abdominal aortic aneurysm development. Eur Heart J. 2020;41(26):2469-2471.

23. Dalman RL, Matthew M. Management of asymptomatic abdominal aortic aneurysm. UpToDate. Sep 27, 2019. https://www.uptodate.com/contents/management-ofasymptomatic-abdominal-aortic-aneurysm.

24. Skibba AA, Evans JR, Hopkins SP, Yoon HR, Katras T, Kalbfleisch JH, Rush DS. Reconsidering gender relative to risk of rupture in the contemporary management of abdominal aortic aneurysms. J Vasc Surg. 2015;62(6):14291436.

25. Dillavou ED. Surgical and endovascular repair of rup- 
tured abdominal aortic aneurysm. UpToDate. March 30, 2020. https://www.uptodate.com/contents/surgical-andendovascular-repair-of-ruptured-abdominal-aortic-aneurysm.

26. Shames ML, Sanchez LA, Rubin BG, Choi ET, Geraghty PJ, Flye MW, Thompson RW, et al. Delayed complications after endovascular AAA repair in women. J Endovasc Ther. 2003;10(1):10-15.

27. Malayala SV, Raza A. Health behavior and perceptions among African American women with metabolic syndrome. J Community Hosp Intern Med Perspect.
2016;6(1):30559.

28. Malayala SV, Raza A. Compliance with USPSTF recommendations on aspirin for prevention of cardiovascular disease in men. Int J Clin Pract. 2016;70(11):898-906.

29. Malayala SV, Raza A. A comparative analysis of cardiovascular risk in human immunodeficiency virus-seropositive and -seronegative pre-menopausal women. J Clin Med Res. 2020;12(7):409-414.

30. Malayala SV, Raza A. A comparative analysis of the 10 year cardiovascular risk in HIV Positive Men and Women. Delaware Medical Journal. 2020:92(2):72-75. 